\title{
Direct emission of multiple strange baryons in ultrarelativistic heavy-ion collisions from the phase boundary
}

\author{
A. Dumitru ${ }^{1}$, S.A. Bass ${ }^{2}$, M. Bleicher ${ }^{3}$, H. Stöcker ${ }^{3}$, W. Greiner ${ }^{3}$ \\ ${ }^{1}$ Physics Department, Yale University, P.O. Box 208124, New Haven, CT 06520, USA \\ ${ }^{2}$ Department of Physics, Duke University, Durham, NC 27708, USA \\ ${ }^{3}$ Institut für Theoretische Physik, J.W. Goethe Universität, Robert-Mayer Str. 10, 60054 Frankfurt, Germany
}

\begin{abstract}
We discuss a model for the space-time evolution of ultrarelativistic heavy-ion collisions which employs relativistic hydrodynamics within one region of the forward light-cone, and microscopic transport theory (i.e. UrQMD) in the complement. Our initial condition consists of a quark-gluon plasma which expands hydrodynamically and hadronizes. After hadronization the solution eventually changes from expansion in local equilibrium to free streaming, as determined selfconsistently by the interaction rates between the hadrons and the local expansion rate. We show that in such a scenario the inverse slopes of the $m_{T}$-spectra of multiple strange baryons $(\Xi, \Omega)$ are practically unaffected by the purely hadronic stage of the reaction, while the flow of $p$ 's and $\Lambda$ 's increases. Moreover, we find that the rather "soft" transverse expansion at RHIC energies (due to a first-order phase transition) is not washed out by strong rescattering in the hadronic stage. The earlier kinetic freeze-out as compared to SPS-energies results in similar inverse slopes (of the $m_{T}$-spectra of the hadrons in the final state) at RHIC and SPS energies.
\end{abstract}

Ultrarelativistic heavy ion collisions offer the unique opportunity to study highly excited QCD-matter in the laboratory, and possibly the QCD phase transition to the so-called quark-gluon plasma (QGP) at high energy density [1]. The dynamics of such reactions is commonly described either within hydrodynamics or within microscopic transport models [2]. The fluid-dynamical approach is most accurate in very dense systems (where mean free paths are small) close to local equilibrium. In this model the reaction dynamics is closely linked to the equation of state $(\mathrm{EoS})$, which enters directly into the equations of motion. It is therefore well suited to study systems that undergo phase transitions, e.g. that of hadronic matter into a QGP. In particular, one can employ an EoS with a first order phase transition, where the QGP coexists with hadronic matter within some region of energy- and baryon density.

Hadronic cascades based on binary hadron-hadron collisions are probably less well suited to study very dense systems, and of hadrons coexisting with a QGP. However, they are useful for the dilute space-time regions since they account for finite relaxation times in the hadron gas 30 and the breakup of local kinetic equilibrium. Moreover, since each hadron is propagated individually, and its interactions with other hadrons are described on the basis of elementary processes, microscopic transport models offer the opportunity to calculate the freeze-out conditions instead of just putting them in by hand as is usually done in fluid-dynamical approaches. In particular, one needs not assume that all hadron species decouple on the same hypersurface [4,5].

The sequencial freeze-out is particularly important to understand [6] the experimental fact that in $\mathrm{Pb}+\mathrm{Pb}$ collisions at CERN-SPS energy $(\sqrt{s}=17 A \mathrm{GeV})$ the transverse mass spectra of multiple strange baryons [7],8] are "softer" than expected by a linear interpolation of the inverse slopes of protons and deuterons [9]: If all hadrons flow with the same collective velocity and decouple on the same hypersurface, the inverse slopes or average transverse momenta have to increase with the mass of the hadron (at least if one neglects resonance decays).

We shall discuss here, whether it is possible to understand these observations by assuming the formation of a QGP which expands as an ideal relativistic fluid, going through a mixed phase where it coexists with hadronic matter. After hadronization is completed (locally in space-time), we evolve the system within microscopic transport theory (we employ the UrQMD model [10]), and follow the evolution of the hadrons until they freeze-out. We point out that the hadrons are "born" in an expanding three-volume (i.e. the hadronization hypersurface), the expansion rate of that volume being many orders of magnitude larger than in the universe at hadronization [11]. It will thus depend on the corresponding elementary cross-sections and the composition of the system which hadrons are able to maintain local kinetic equilibrium, despite the very large expansion rate. We will show in particular how the momentum distributions of various hadron species are affected by the hadronic stage as compared to those on the hadronization hypersurface.

Let us first briefly describe the specific form of hydrodynamics that we shall employ. For a more detailed discussion see 12,13. For simplicity, we assume boost-invariant longitudinal flow, $v_{z}=z / t$. This should be a reasonable first approximation around midrapidity. Cylindrically symmetric transverse expansion is superimposed. For $T>T_{C}=160 \mathrm{MeV}$ we employ the wellknown MIT bagmodel equation of state, assuming for simplicity an ideal gas of quarks, antiquarks (with masses $m_{u}=m_{d}=0, m_{s}=150 \mathrm{MeV}$ ), and gluons. For $T<T_{C}$ we employ an ideal hadron gas that includes the complete hadronic spectrum up to a mass of $2 \mathrm{GeV}$. At $T=T_{C}$ 
we require that both pressures are equal, which fixes the bag constant to $B=380 \mathrm{MeV} / \mathrm{fm}^{3}$. By construction the EoS exhibits a first-order phase transition.

For collisions at SPS energy we assume that hydrodynamic flow sets in on the proper time hyperbola $\tau_{i}=$ $1 \mathrm{fm} / \mathrm{c}$. This is a value conventionally assumed in the literature, cf. e.g. [12,13]. We further employ a (net) baryon rapidity density (at midrapidity) of $d N_{B} / d y=80$, as obtained by the NA49-collaboration for central $\mathrm{Pb}+\mathrm{Pb}$ reactions [14]. The average specific entropy in these collisions is $\bar{s} / \bar{\rho}_{B}=45 \pm 5$ (the bar means averaging over the transverse plane). With this entropy per net baryon most measured hadron multiplicity ratios can be described within $\pm 20 \%$ [15]. The corresponding initial energy and net baryon densities $\left(\bar{\epsilon}_{i}=6.1 \mathrm{GeV} / \mathrm{fm}^{3}, \bar{\rho}_{i}=4.5 \rho_{0}\right)$ are assumed to be distributed in the transverse plane according to a so-called "wounded nucleon" distribution with transverse radius $R_{T}=6 \mathrm{fm}$ [13].

The measured $p_{T}$-spectra (at midrapidity) of $\pi$ 's, $p$ 's, $\Lambda$ 's, $\phi$ 's, and $d$ 's can be reproduced within this model if one assumes an ideal hydrodynamic expansion between the $\tau=\tau_{i}$ hyperbola and the $T=130 \mathrm{MeV}$ isotherm [13]. However, the resulting spectra of $\Xi$ 's and $\Omega$ 's are harder than those of $p$ 's and $\Lambda$ 's, because the same freeze-out hypersurface is employed for all hadrons.

Due to the higher parton density at midrapidity (as compared to collisions at SPS energy), thermalization may be reached earlier at RHIC. According to various studies [16], it may occur within $0.2-1 \mathrm{fm}$. We employ $\tau_{i}=0.6 \mathrm{fm}$. The net baryon rapidity density and specific entropy at midrapidity in central $\mathrm{Au}+\mathrm{Au}$ at $\sqrt{s}=200 A \mathrm{GeV}$ is predicted by various models of the initial evolution to be in the range $d N_{B} / d y \approx 20-35$, $s / \rho_{B} \approx 150-250$ [17]. We will employ $d N_{B} / d y=25$ and $\bar{s} / \bar{\rho}_{B}=205\left(\rightarrow \bar{\epsilon}_{i}=20 \mathrm{GeV} / \mathrm{fm}^{3}, \bar{\rho}_{i}=2.3 \rho_{0}\right)$. (The initial conditions could of course be fine-tuned once the first experimental data are available.) This yields a transverse energy on the $\tau=\tau_{i}$ hyperbola of $d E_{T} / d y=1.3 \mathrm{TeV}$, which decreases to $720 \mathrm{GeV}$ on the hadronization hypersurface. We find that $d E_{T} / d y$ is almost conserved during the following hadronic evolution, i.e. dissipation compensates for the expansion work.

Having specified the initial conditions and the EoS, the hydrodynamical solution between the $\tau=\tau_{i}$ hyperbola and the hadronization hypersurface is uniquely determined. For a more detailed discussion of how this solution is obtained, cf. [13].

The number of hadrons of species $i$ hadronizing at space-time rapidity $\eta$, proper time $\tau$, and position $r_{T}(\sin (\chi-\phi), \cos (\chi-\phi))$, with four-momentum $p^{\mu}=$ $\left(m_{T} \cosh y, p_{T} \sin \chi, p_{T} \cos \chi, m_{T} \sinh y\right)$, is given by [18]

$$
\begin{aligned}
\frac{d N_{i}}{d^{2} m_{T} d y d \eta d \zeta d \phi} & =r_{T} \tau\left(p_{T} \cos \phi \frac{d \tau}{d \zeta}\right. \\
& \left.-m_{T} \cosh (y-\eta) \frac{d r_{T}}{d \zeta}\right) f(p \cdot u)
\end{aligned}
$$

where $u^{\mu}=\gamma_{T}\left(\cosh \eta, v_{T} \sin (\chi-\phi), v_{T} \cos (\chi-\phi), \sinh \eta\right)$ denotes the fluid four-velocity. $\zeta \in[0,1]$ parametrizes the hadronization hypersurface (counter-clockwise), such that $r_{T}(\zeta)$ and $\tau(\zeta)$ specify the space-time points on the hypersurface. $f$ is either a Bose-Einstein or Fermi-Dirac distribution function. We assume that $v_{T}, T$, and the chemical potentials, are independent of $\eta$, such that the hadronization hypersurface in the $t-z$ plane $\left(\right.$ fixed $r_{T}$ ) is simply a proper-time hyperbola.

The ensemble of hadrons generated according to eq. (0.1) is now taken as input for the microscopic transport model UrQMD. Thus, after formation each hadron is propagated individually along a classical trajectory and can scatter stochastically until it eventually does not interact any more and freezes out. Note that by construction the hadron fluid starts from a state of local equilibrium, but in a three-space with very rapidly increasing volume-measure [11]. The energy-momentum tensors and conserved currents (of UrQMD and hydrodynamics) match on the hadronization hypersurface, because the local collective flow velocities, as well as the energy and net baryon densities, and the pressures, are equal (we include the same states in the hadronic part of the fluiddynamical EoS as in UrQMD).

The UrQMD model is based on the covariant propagation of all hadrons, excitation of resonances and strings and their subsequent decay resp. fragmentation. Free cross-sections for hadron-hadron scattering are employed. Comparisons of UrQMD calculations to various experimental data from SIS to SPS energies, as well as a detailed description of the model, are documented elsewhere [10].

Fig. 11 compares the $m_{T}$-spectra on the hadronization hypersurface (thin lines), obtained from Eq. (0.1) (plus strong resonance decays), with those at freeze-out (thick lines). The $(+)-(-)[19], \Lambda, \bar{\Lambda}[20$, and $\Xi$ [8] spectra of NA49, and the $\Lambda, \Lambda, \Xi$, and $\Omega$ spectra of WA97 [7] are also depicted (the latter have been normalized to our calculation). Clearly, because of rescattering, the transverse flow of $N$ 's and $\Lambda$ 's increases during the hadronic stage. Also, slow antinucleons (with $m_{T}<1.5 \mathrm{GeV}$ ) are annihilated to a large extent because the annihilation cross-section increases rapidly at small momenta. On the other hand, the spectra of $\Omega$ 's and of $\Xi$ 's with $m_{T}>1.6 \mathrm{GeV}$ are practically unaffected by the hadronic stage and closely resemble those on the phase boundary. This is due to the fact that the scattering rates of $\Xi$ and $\Omega$ in a pion-rich hadron gas are smaller than those of $N$ 's and $\Lambda$ 's $[$ [ . Note that a $\pi$ and $\Omega$ can not be coupled to any of the known 21] $B=1, S=-3$ states with masses

\footnotetext{
${ }^{1}$ The switch is actually done slightly behind the hadronization hypersurface, cf. the discussion in 13], because the rate of expansion of its volume can diverge [11].
} 
$<2.5 \mathrm{GeV}$. The excitation of a state above this threshold requires a large $(\Delta E \sim 1 \mathrm{GeV})$ energy transfer from the $\pi$ to the $\Omega$ (in the local rest-frame), which is highly suppressed since on the hadronization hypersurface the $\pi$-energies are distributed thermally with $\langle E\rangle_{\pi} \approx 3 T_{C}$. Finally, elastic collisions give much smaller energy and momentum transfers to the $\Omega$ than $m_{\Omega}$, and therefore do not change its $m_{T}$-distribution.

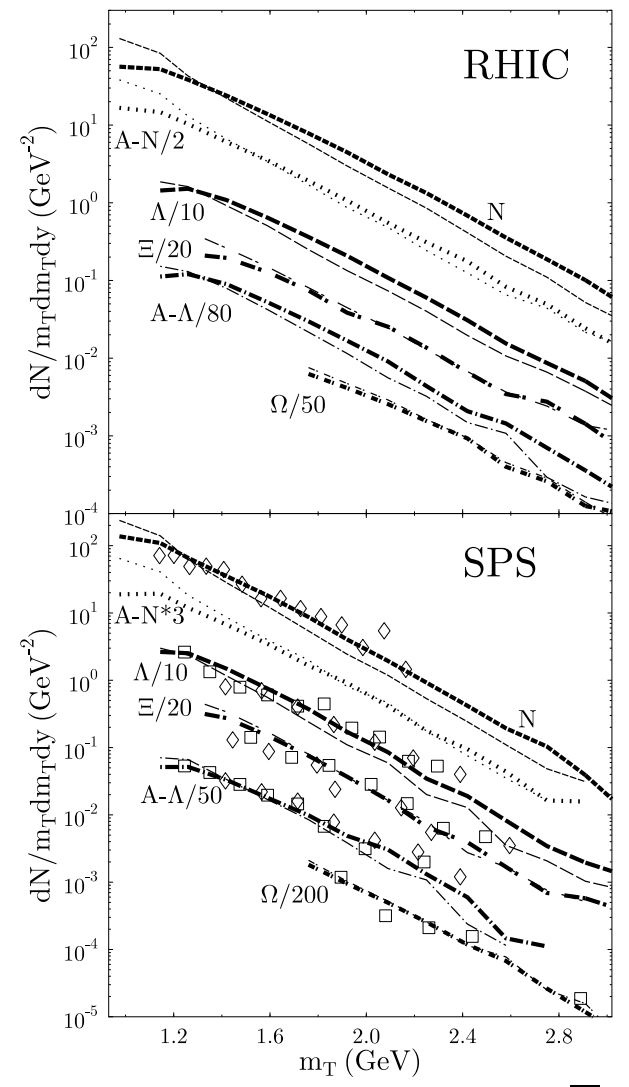

FIG. 1. Transverse mass spectra of $N, \bar{N}, \Lambda+\Sigma^{0}$, $\Xi^{0}+\Xi^{-}$, and $\Omega^{-}$at $y_{\text {c.m. }}=0$ in central $\mathrm{Pb}+\mathrm{Pb}$ collisions at $\sqrt{s}=17 A \mathrm{GeV}$ (lower panel) and $\mathrm{Au}+\mathrm{Au}$ at $\sqrt{s}=200 A \mathrm{GeV}$ (upper panel). Thin lines: on the hadronization hypersurface; thick lines: at freeze-out; diamonds: NA49 data; squares: WA97 data.

As shown in Fig. 2, on average the baryons which finally emerge as $\Xi$ 's and $\Omega$ 's suffer even less interactions than the final-state $\bar{p}$ 's and $\bar{\Lambda}$ 's. Thus, within the model presented here, these particles are emitted directly from the hadronization hypersurface without further rescattering in the hadronic stage. The hadron gas emerging from the hadronization of the QGP (in these high-energy reactions) is almost "transparent" for the multiple strange baryons, especially because of the large expansion rate. In our calculation, the flow seen in the $\Omega$-spectra at SPS and RHIC is fully accounted for by the expansion preceeding hadronization (for entropy and baryon density as discussed above). The space-time domains of freeze- out for several hadron species are discussed in 22].

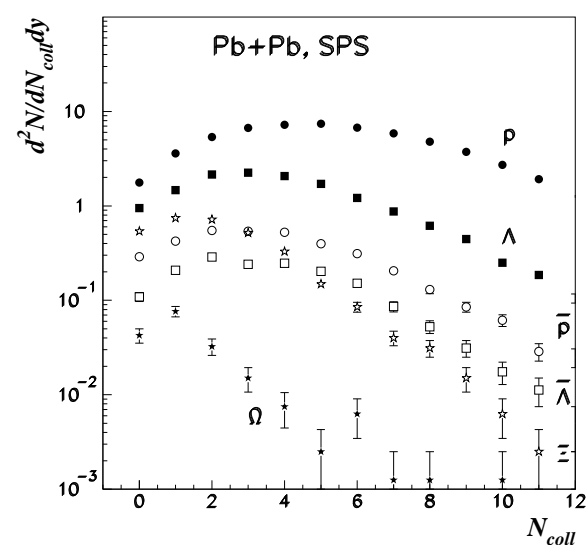

FIG. 2. Distribution of the number of interactions that the final-state particles suffer after being hadronized.

Fig. 3 displays the inverse slopes $T^{*}$ obtained by a fit of $d N_{i} / d^{2} m_{T} d y$ to $\exp \left(-m_{T} / T^{*}\right)$ in the range $m_{T}-m_{i}<1 \mathrm{GeV}$ (for clarity, the calculated points have been slightly displaced to the left or right of the true hadron mass). The trend of the SPS data, namely the "softer" spectra of $\Xi$ 's and $\Omega$ 's as compared to a linear $T^{*}(m)$ relation, is reproduced reasonably well. This is in contrast to "pure" hydrodynamics with kinetic freezeout on a common hypersurface (e.g. the $T=130 \mathrm{MeV}$ isotherm), where the stiffness of the spectra increases with mass, cf. Fig. 3 and also refs. [5, 13, 23]. Resonance decays are not included in the hydrodynamic spectra on the $T=130 \mathrm{MeV}$ isotherm.

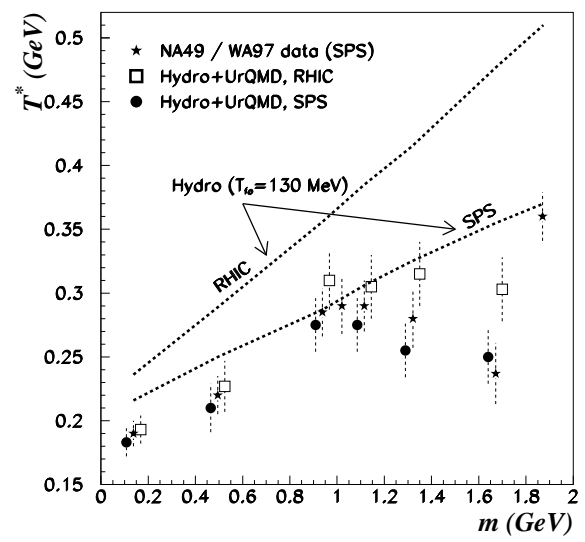

FIG. 3. Inverse slopes of the $m_{T}$-spectra of $\pi, K, p, \Lambda+\Sigma^{0}$, $\Xi^{0}+\Xi^{-}$, and $\Omega^{-}$at $y_{\text {c.m. }}=0, m_{T}-m_{i}<1 \mathrm{GeV}$.

When going from SPS to RHIC energy, the model discussed here generally yields only a slight increase of the inverse slopes, although the specific entropy is larger by a factor of 4-5! The reason for this behavior is the firstorder phase transition that leads to smaller transverse flow velocities than an ideal gas [24] (but to a larger expansion rate of the hadronization volume [11] !). For 
our initial conditions, the collective transverse flow velocity on the hadronization hypersurface increases only from $\approx 0.3(\mathrm{~Pb}+\mathrm{Pb}$ at $\mathrm{SPS})$ to $\approx 0.35(\mathrm{Au}+\mathrm{Au}$ at RHIC) [13]. As can be seen from the present calculation, this is not counterbalanced by increased rescattering in the purely hadronic stage (compare to the inverse slopes obtained from "pure" hydrodynamics with freeze-out on the $T=130 \mathrm{MeV}$ isotherm !).

In summary, we have employed relativistic hydrodynamics to describe the evolution and hadronization of a hypothetical quark-gluon fluid at CERN-SPS and BNLRHIC energies. The produced hadrons are propagated within a microscopic transport model (UrQMD). Interactions within the hadron gas increase the collective flow beyond that present at hadronization, and reduce the temperature below the QCD phase transition temperature (we assume $T_{C}=160 \mathrm{MeV}$ ). As an exception, we find that multiple strange baryons practically do not rescatter within the hadron gas. Their $m_{T}$-spectra are thus determined by the conditions on the hadronization hypersurface, i.e. $T_{C}$ and the collective flow created prior to hadronization. Their spectra therefore are less sensitive to the confined phase, $T<T_{C}$, but are closely related to the EoS of the QGP and the phase transition temperature $T_{C}$. At RHIC energy, for all hadrons $T^{*}$ or $\left\langle p_{T}\right\rangle$ are considerably smaller than predicted by pure hydrodynamics with freeze-out on the $T=130 \mathrm{MeV}$ isotherm. Thus, kinetic freeze-out occurs closer to the phase boundary than at SPS energy; this is due to the different chemical composition of the central region (more mesons, less baryons) and due to the larger expansion rate of the hadronization volume.

\section{ACKNOWLEDGMENTS}

We thank T. Ullrich and N. Xu for helpfull comments. A.D. gratefully acknowledges a fellowship by the German Academic Exchange Service (DAAD). S.A.B. is supported in part by the Alexander von Humboldt Foundation, and by DOE grant DE-FG02-96ER40945. M.B. thanks the Josef Buchmann Foundation for support. This work has been supported in part by BMBF, DFG, and GSI.

[1] E.V. Shuryak, Phys. Rept. 61, 71 (1980); L. McLerran, Rev. Mod. Phys. 58, 1021 (1986); B. Müller, Rept. Prog. Phys. 58, 611 (1995); S.A. Bass, M. Gyulassy, H. Stöcker, and W. Greiner, J. Phys. G 25, R1 (1999)

[2] H. Stöcker and W. Greiner, Phys. Rept. 137, 277 (1986); R.B. Clare and D. Strottman, Phys. Rept. 141, 177 (1986)

[3] P. Danielewicz, Phys. Lett. B 146, 168 (1984); P. Levai and B. Müller, Phys. Rev. Lett. 67, 1519 (1991); M.
Prakash, M. Prakash, R. Venugopalan, and G. Welke, Phys. Rept. 227, 321 (1993)

[4] L.V. Bravina, I.N. Mishustin, N.S. Amelin, J.P. Bondorf, and L.P. Csernai, Phys. Lett. B 354, 196 (1995); H. Sorge, Phys. Lett. B 373, 16 (1996); S. Pratt and J. Murray, Phys. Rev. C 57, 1907 (1998)

[5] C.M. Hung and E. Shuryak, Phys. Rev. C 57, 1891 (1998)

[6] H. van Hecke, H. Sorge, and N. Xu, Phys. Rev. Lett. 58, 5764 (1998)

[7] E. Andersen et al. (WA97 Collaboration), Phys. Lett. B 433, 209 (1998)

[8] H. Appelshäuser et al., (NA49 collaboration), Phys. Lett. B 444, 523 (1998)

[9] I.G. Bearden et al., (NA44 Collab.), Phys. Rev. Lett. 78, 2080 (1997)

[10] S.A. Bass et al., Prog. Part. Nucl. Phys. 41, 225 (1998)

[11] A. Dumitru, hep-ph/9905217

[12] J.D. Bjorken, Phys. Rev. D 27, 140 (1983); K. Kajantie and L. McLerran, Nucl. Phys. B214, 261 (1983)

[13] A. Dumitru and D.H. Rischke, Phys. Rev. C 59, 354 (1999)

[14] H. Appelshäuser et al., (NA49 collaboration), Phys. Rev. Lett. 82, 2471 (1999)

[15] J. Letessier, A. Tounsi, U. Heinz, J. Sollfrank, and J. Rafelski, Phys. Rev. Lett. 70, 3530 (1993); P. BraunMunzinger, J. Stachel, J.P. Wessels, and N. Xu, Phys. Lett. B 365, 1 (1996); C. Spieles, H. Stöcker, and C. Greiner, Eur. Phys. J. C2, 351 (1998); M. Reiter, A. Dumitru, J. Brachmann, J.A. Maruhn, H. Stöcker, and W. Greiner, Nucl. Phys. A643, 99 (1998)

[16] B. Müller and X.N. Wang, Phys. Rev. Lett. 68, 2437 (1992); E. Shuryak, Phys. Rev. Lett. 68, 3270 (1992); K. Geiger, Phys. Rev. D 46, 4965 (1992); T.S. Biro, E. van Doorn, B. Müller, M.H. Thoma, and X.N. Wang, Phys. Rev. C 48, 1275 (1993); B. Kämpfer and O.P. Pavlenko, Z. Phys. C 62, 491 (1994); K.J. Eskola and X.N. Wang, Phys. Rev. D 49, 1284 (1994); K.J. Eskola and K. Kajantie, Z. Phys. C 75, 515 (1997)

[17] K. Geiger and J.I. Kapusta, Phys. Rev. D 47, 4905 (1993); T. Schönfeld, H. Stöcker, W. Greiner, and H. Sorge, Mod. Phys. Lett. A 8, 2631 (1993); L. Gerland et al., nucl-th/9512032; S.E. Vance, M. Gyulassy, and X.N. Wang, Nucl. Phys. A638, 395c (1998)

[18] F. Cooper and G. Frye, Phys. Rev. D 10, 186 (1974)

[19] P.G. Jones and the NA49 collaboration, Nucl. Phys. A610, 188c (1996);

[20] C. Bormann et al., (NA49 collaboration), J. Phys. G 23, 1817 (1997)

[21] C. Caso et al., Eur. Phys. J. C3, 1 (1998)

[22] S.A. Bass et al., nucl-th/9902062

[23] H. Stöcker, A.A. Ogloblin, and W. Greiner, Z. Phys. A 303, 259 (1981); T. Csörgö and B. Lörstad, Phys. Rev. C 54, 1390 (1996); B. Kämpfer, O.P. Pavlenko, A. Peshier, M. Hentschel, and G. Soff, J. Phys. G 23, 2001 (1997)

[24] M. Kataja, P.V. Ruuskanen, L.D. McLerran, and H. von Gersdorff, Phys. Rev. D 34, 2755 (1986); C.M. Hung and E. Shuryak, Phys. Rev. Lett. 75, 4003 (1995); D.H. Rischke and M. Gyulassy, Nucl. Phys. A597, 701 (1996); Nucl. Phys. A608, 479 (1996) 